\title{
Magnetic reconnection exhausts at the boundaries of small interplanetary magnetic flux ropes
}

\author{
H. Q. Feng ${ }^{1,2}$, D. J. Wu ${ }^{3}$, J. M. Wang ${ }^{1}$, and J. W. Chao ${ }^{4}$ \\ 1 College of Physics and Electronic Information, Luoyang Normal University, Luoyang 471022, PR China \\ e-mail: fenghq9921@163.com \\ 2 State Key Laboratory of Space Weather, Center for Space Science and Applied Research, Chinese Academy of Sciences, \\ Beijing 100190, PR China \\ 3 Purple Mountain Observatory, Chinese Academy of Sciences, Nanjing 210008, PR China \\ 4 Institute of Space Science, National Central University, Chungli 32001, Taiwan
}

Received 21 March 2010 / Accepted 15 December 2010

\section{ABSTRACT}

Context. Small interplanetary magnetic flux ropes (SIMFRs) are commonly observed by spacecraft at 1 AU. Aims. We provide a mechanism to decrease the dimensions of some SIMFRs as they propagate away from the Sun. Methods. We carefully examined the high-resolution magnetic field and plasma data from the Wind spacecraft during the period 1995-2005 to identify X-line magnetic reconnection exhausts at the boundaries of SIMFRs.

Results. We identified nine X-line magnetic reconnection exhausts at the boundaries of SIMFRs, which were destroying the flux within the related SIMFRs.

Conclusions. These observational facts indicate that the boundaries of some SIMFRs were still evolving through interaction with the background solar wind, and their spatial scales would diminish gradually.

Key words. solar-terrestrial relations - solar wind

\section{Introduction}

The interplanetary magnetic flux rope (IMFR) is an important solar wind magnetic structure with a diameter distribution in the range of 0.0039-0.6266 AU (Moldwin et al. 1995, 2000; Feng et al. 2007). Among IMFRs, the larger-scale-structure magnetic clouds (MCs) often have diameters exceeding 0.20 AU near the Earth (e.g., Goldstein 1983; Burlaga 1988; Lepping et al. 1990; Farrugia et al. 1995). Magnetic clouds are a subset of interplanetary coronal mass ejections (ICMEs), which are interplanetary manifestations of transient events with large amounts of material ejected from the solar atmosphere (e.g., Hundhausen 1987; Kahler 1987). The outstanding characteristics of MCs are enhanced magnetic field strength, low proton temperature, low plasma beta value, and a smooth rotation in the direction of the magnetic field (Burlaga et al. 1990). In the past decade, a number of studies have been conducted on MCs, focusing on the magnetic and plasma structures, its solar origins, and the induced geomagnetic activity (e.g., Bothmer \& Schwenn 1998; Webb et al. 2000; Wu \& Lepping 2002). In comparison, only a few studies have been conducted on small interplanetary magnetic flux ropes (SIMFRs), and their origin still remains disputed (e.g., Moldwin et al. 2000; Feng et al. 2007, 2008; Cartwright \& Moldwin 2008; Feng \& Wu 2009). Moldwin et al. $(1995,2000)$ first reported several SIMFRs, suggesting that these SIMFRs do not originate from the coronal region, and that instead the SIMFRs may be a result of the magnetic reconnection at the heliospheric current sheet. The main piece of evidence corroborating this argument is the lack of intermediate-sized events (durations of several hours). If SIMFRs and MCs are the same phenomenon, the size distribution should be continuous. Recently,
Feng et al. (2007) systematically surveyed the solar wind data of the Wind spacecraft from 1995 to 2001 and found that IMFRs have a continuous size distribution. Their analysis of IMFRs with different scales showed that the physical properties of the IMFRs changed slowly with their increasing scale sizes. Therefore, Feng et al. (2007) suggested that SIMFRs also originate in the solar corona and are interplanetary manifestations of small CMEs produced in small solar eruptions.

Magnetic reconnection is a process that converts magnetic energy into bulk flow energy. This process generally favors oppositely directed magnetic fields at thin current sheets and plays an important role in many interpretations and models of space, solar, astrophysical, and laboratory plasma phenomena. Simulation results show that multiple X-line magnetic reconnections can produce the rope topology (Lee \& Fu 1985; Lee et al. 1993). Wei et al. (2003) found that most MCs have boundary layers displaying a drop in magnetic field magnitude and a significant change in field direction. These boundary layers also have properties of high proton temperature, density, and plasma beta. Wei et al. (2003) argued that these boundary layers can form through the magnetic reconnection process as a result of the interactions between the MCs and the ambient medium, and the dimensions of IMFRs can decrease owing to the magnetic reconnections near their boundaries as they propagate away from the Sun. However, Wei et al. (2003) did not provide any direct evidence of reconnection in the boundary layers. Recently, many in-situ solar wind reconnection events have been reported (e.g., Gosling et al. 2005) and SIMFRs have also been frequently encountered by spacecraft (e.g., Moldwin et al. 1995, 2000; Feng et al. 2007, 2008; Cartwright \& Moldwin 2008). It is very desirable to understand whether these reported SIMFRs are related 


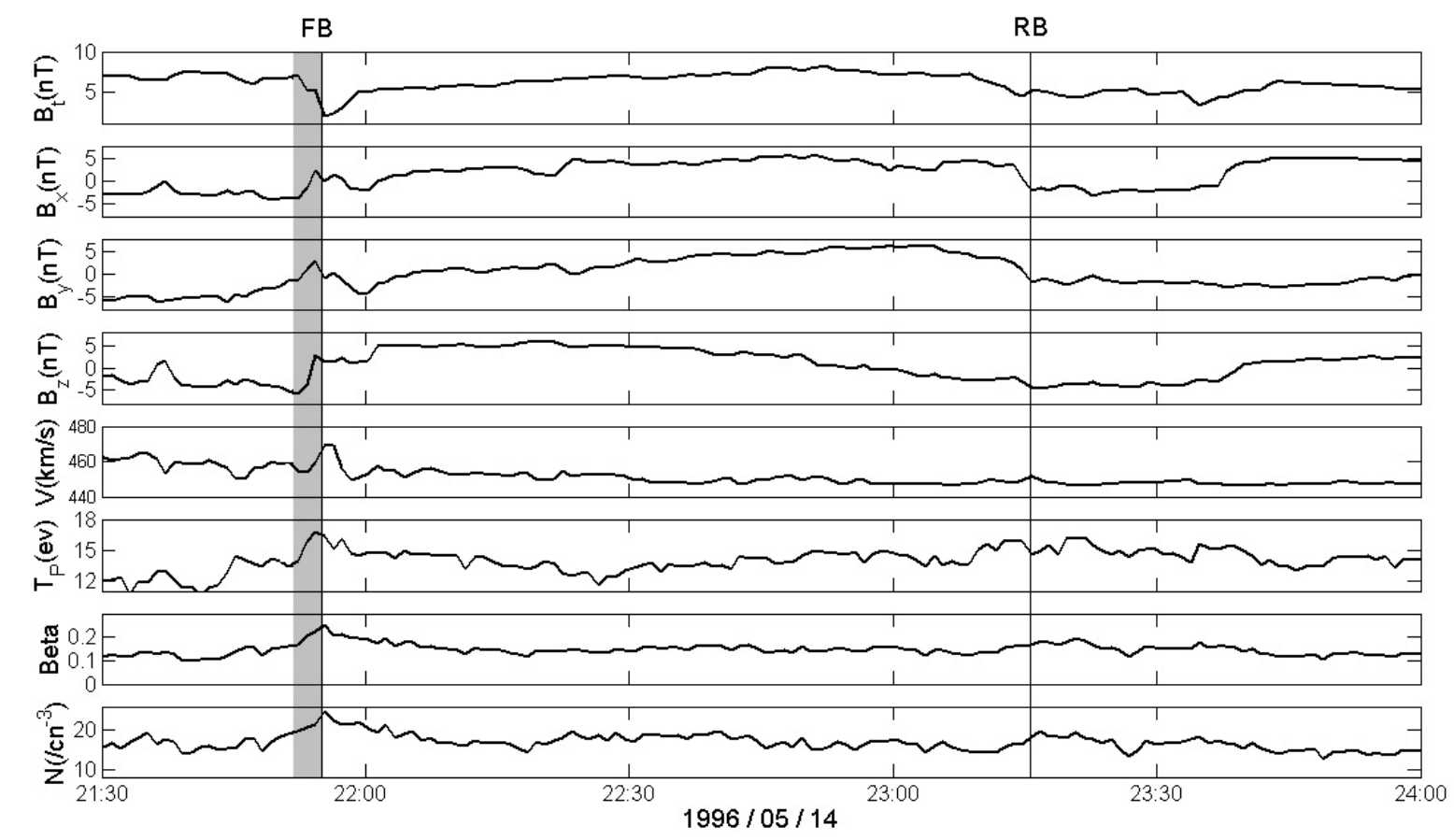

Fig. 1. Interplanetary magnetic field and plasma data measured by the Wind spacecraft on 1996 May 14. The gray shaded region (21:53:0521:53:58 UT) indicates the magnetic reconnection exhaust. FB and RB are the front and rear boundaries of the interplanetary magnetic flux rope, respectively.

to the reported reconnection events. Recently, we reported a SIMFR juxtaposed with a reconnection exhaust, which was observed by Wind and ACE on 1998 Mar. 25. The trailing boundary of the SIMFR was just the leading edge of the X-line reconnection exhaust reported by Gosling et al. (2005), which is to say that there is no measurable separation between them. This means that reconnection was opening the flux rope fields instead of generating the small magnetic flux rope (Feng \& Wu 2009). Therefore, the spatial scale of the flux rope would diminish gradually. In addition, Gosling et al. (2007) have reported reconnection exhausts observed at the edges of large flux ropes in the solar wind. These observations provided some evidence that the dimensions of IMFRs can be decreased by the magnetic reconnections near their boundaries. Moreover, Gosling et al. (2007) also reported reconnection exhausts within the interiors of IMFRsin contrast to the cases at the boundary of IMFRs. They observed some events at the thin current sheets with considerably less local magnetic shear angles. However, the reported SIMFR related with a magnetic reconnection is merely an isolated example. In this paper, the relationship between SIMFRs and magnetic reconnection exhausts is studied in greater detail. Our purpose is to investigate if even more SIMFRs were being destroyed by their related magnetic reconnections exhausts.

\section{Data and method of analysis}

Cartwright \& Moldwin (2008) and Feng et al. (2008) recently presented a comprehensive survey of SIMFRs during the period 1995-2005. Cartwright \& Moldwin (2008) identified 68 SIMFRs using one-minute averaged plasma and magnetic field data from Wind. Using the same data, Feng et al. (2008) identified 125 SIMFRs. The present study is based on the SIMFR lists of Cartwright \& Moldwin (2008) and Feng et al. (2008). However, Feng et al. (2008) misidentified many Alfvénic rotations as SIMFRs from their list (Cartwright \& Moldwin 2008), and several SIMFRs from Cartwright \& Moldwin's (2008) list have no apparent flux rope signature. Therefore, we re-examined the SIMFR lists of Cartwright \& Moldwin (2008) and Feng et al. (2008). In the Cartwright \& Moldwin (2008) database, we found that 10 of the 68 events do not have the essential property of a smooth rotation in the magnetic direction, and three events show significant fluctuations during the rotations. We believe that these 10 events were not magnetic flux ropes, and the other three events do not seem to be magnetic flux ropes either. Even though some events did not show perfectly smooth rotations, they are considered flux ropes because they satisfy the criteria of IMFRs(Feng et al. 2008, 2010). Feng et al. (2010) identified SIMFRs through the following steps: "(1) the candidate of SIMFRs are selected by identifying the rotation of the magnetic field direction and the enhanced magnetic field strength (compared with the ambient medium) by eye; (2) the correlation coefficient of the velocity and magnetic field components is then examined to exclude possible Alfvén wave structures. If two of the field and velocity components have correlation coefficients greater than or equal to 0.6, they are considered possible Alfvén wave structures (Coleman 1967, 1968; Belcher \& Davis 1971; Burlaga \& Turner 1976); (3) finally, the geometric parameter of the flux rope is used to identify the possible events, namely, the magnetic field variation that can be fitted to the cylindrical constant-alpha force-free field. When the difference between the model geometry and the observation data is larger than the criterion value $\left(\sqrt{\chi^{2}}=0.3\right)$, the case is not considered a flux rope". The deviation can be expressed by the minimum chi-square $\chi^{2}$ (see Eq. (2) of Feng et al. 2008 for a detailed description of the fitting method). In the Feng et al. (2008) SIMFR list, 77 possible Alfvén wave events are excluded. In addition, five SIMFRs overlap in the lists of Cartwright \& Moldwin (2008) and Feng et al. (2008). In summary, we selected only 98 SIMFRs for our database by examining and identifying the magnetic reconnection exhausts at their boundaries. 


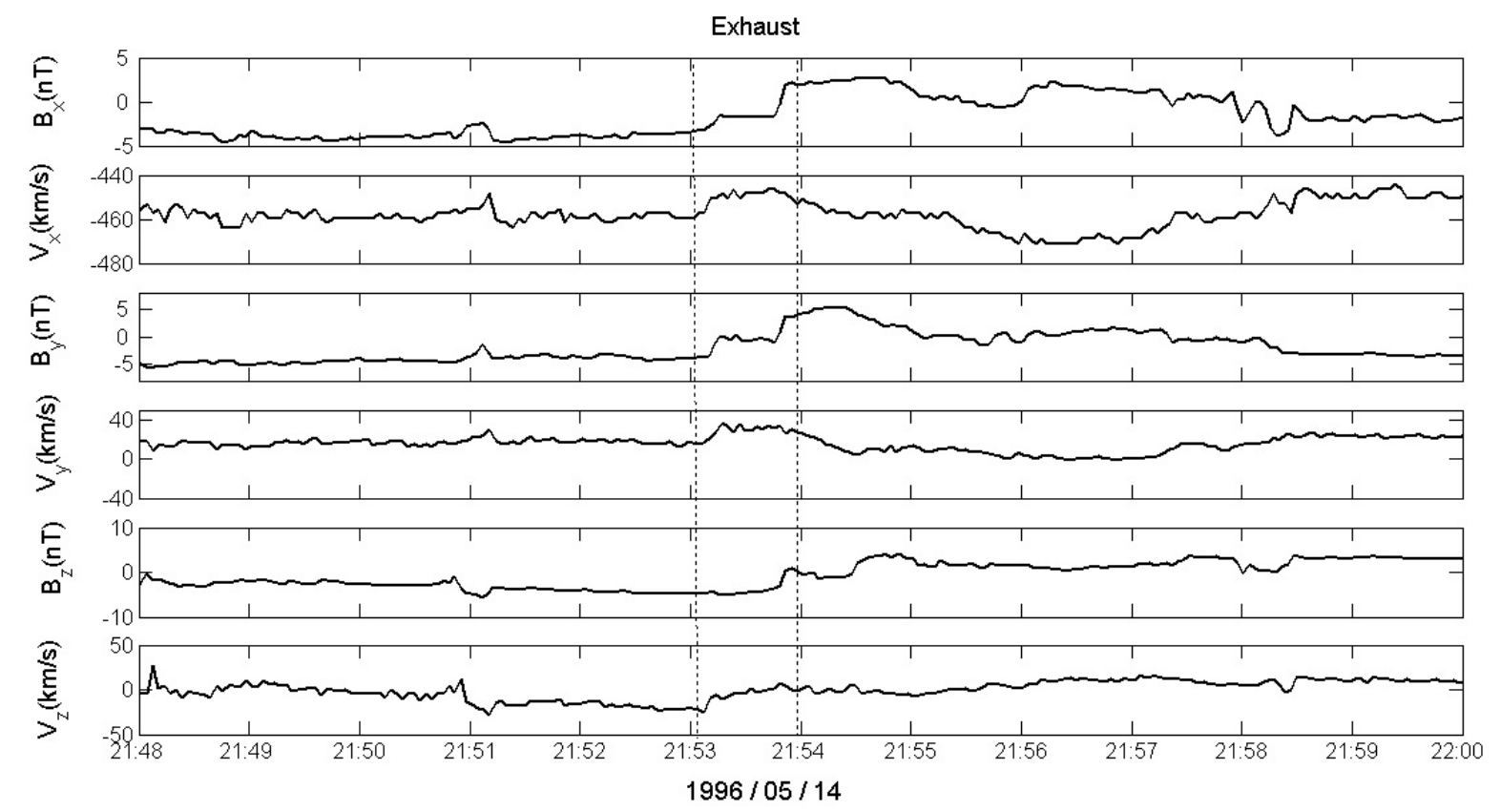

Fig. 2. Interplanetary magnetic field and speed data measured by the Wind spacecraft during the 1996 May 14 magnetic reconnection exhaust passage. The dotted vertical lines indicate the beginning and the end of the reconnection exhaust.

Recently Gosling et al. (2005) recently introduced magnetic reconnection exhaust criteria based on the Petschek reconnection model (Petschek 1964). The exhausts are roughly Alfvénic accelerated or decelerated plasma flows confined to magnetic field reorientation regions, which usually take the form of bifurcated (i.e., double step) current sheets. The exhausts are identified using the following characteristics (Gosling et al. 2005): "the changes in the velocity and magnetic field components are anticorrelated on one side and correlated on the other side; within the exhaust the proton beta, the proton temperature and density are often higher than outside; the magnetic field strength is weaker than that of the surrounding solar wind. In addition, although it is relatively common to observe proton density and temperature enhancements and magnetic field strength decreases within exhausts, many exhausts do not exhibit one or more of those characteristics. Almost all the reported reconnection exhausts are observed in low $(<1)$ proton beta solar wind, and the double-step nature of the field rotation is an almost universal feature (occasionally, one or the other of the two steps is not particularly sharp)". The observations of reconnection exhausts revealed that their durations vary from several seconds to more than twenty minutes (Gosling et al. 2007), and their local field shear angles vary from several degrees to very large degrees (near 180 degrees).

\section{Results and discussion}

According to the magnetic reconnection exhaust criteria used by Gosling et al. (2005), the high-resolution (3 s) magnetic field and plasma data from Wind were examined, and nine magnetic reconnection exhausts were identified at the boundaries of nine SIMFRs. Table 1 lists the identified magnetic reconnection exhausts and their related SIMFRs. The first and second columns show the front and the rear boundaries of the SIMFRs, respectively, and the third and fourth columns list the start and end times of the magnetic reconnection exhausts. As shown in Table 1, six magnetic reconnection exhausts occurred at the front boundaries of their related SIMFRs. As an example, Fig. 1 shows the magnetic field and plasma data of the magnetic reconnection exhaust as well as the following SIMFR on 1996 May 14. The magnetic reconnection exhaust is in the shaded region. The magnitude of the total magnetic field $\left(B_{t}\right)$; the $x, y, z$ components of the magnetic field $\left(B_{x}, B_{y}, B_{z}\right)$; the proton speed $(V)$; the proton temperature $\left(T_{\mathrm{P}}\right)$; the proton beta; and the proton density $(N)$ are shown from the top to the bottom of the panels. The coordinate system is the geocentric solar ecliptic (GSE) Cartesian system. In addition, Fig. 2 also provides expanded views of the magnetic field and velocity curves of the magnetic reconnection exhaust. Figures 1 and 2 illustrate the following: (1) the decelerated flow event is within 21:53:05 21:53:58 UT; (2) the decelerated flow event straddled a large $\left(122^{\circ}\right)$ change in the orientation of $\boldsymbol{B}$; (3) the changes in $\boldsymbol{V}$ and $\boldsymbol{B}$ are correlated with one another at the front boundary of the event and are anticorrelated at the rear boundary; (4) the exhaust is characterized by increases in proton density and proton beta; (5) the magnetic field strength is weaker than that of the surrounding solar wind; (6) the ambient solar wind has a low proton beta $(<0.3)$. These features satisfy the criteria of the reconnection exhausts (Gosling et al. 2005). In addition, the background solar wind flows have different plasma and magnetic field characteristics before and after the exhaust event.

As previously mentioned, Fig. 1 also shows the subsequent SIMFR, which was first identified by Cartwright \& Moldwin (2008). From Fig. 1 it is easy to see that the SIMFR displays a smooth rotation of the magnetic field direction and enhanced magnetic field magnitude. One can also find that the bipolar field appears in the $B_{z}$ component, and the core field appears in the $B_{x}$ and $B_{y}$ components. These are all classic flux rope signatures. To obtain the axial direction of this SIMFR, the constant $\alpha$ force-free model was fitted to the observed magnetic fields. A detailed description of the fitting method can be found in Feng et al.'s study $(2007,2008)$. The model fitting results reveal that the axial direction is $\left(\theta=-20^{\circ}, \phi=224^{\circ}\right)$, where $\theta$ and $\phi$ are the latitude and longitude with respect to the ecliptic plane. According 
A\&A 527, A67 (2011)

Table 1. Small interplanetary flux ropes and reconnection exhausts identified in the Wind data.

\begin{tabular}{|c|c|c|c|c|c|c|c|}
\hline No. ${ }^{a}$ & $\mathrm{FB}^{b}$ & $\mathrm{RB}^{c}$ & $\operatorname{Start}^{d}$ & End $^{e}$ & Angle $^{f}$ & $\beta_{1}^{g}$ & $\beta_{2}^{h}$ \\
\hline 01 & 1995-09-20 13:00:30 & 1995-09-20 14:14:15 & 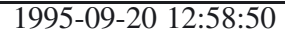 & 1995-09-20 13:00:05 & 154 & 0.16 & 0.29 \\
\hline 02 & $1996-05-14$ 21:53:08 & 1996-05-14 23:34:15 & $1996-05-14$ 21:53:05 & $1996-05-14$ 21:53:58 & 122 & 0.16 & 0.25 \\
\hline 03 & 1996-06-20 16:41:31 & 1996-06-20 18:46:15 & $1996-06-2016: 39: 58$ & 1996-06-20 16:41:31 & 84 & 0.09 & 0.10 \\
\hline 04 & 1996-08-06 00:05:00 & 1996-08-06 03:35:00 & 1996-08-06 00:03:56 & 1996-08-06 00:05:00 & 80 & 0.06 & 0.08 \\
\hline 05 & 1998-03-25 14:08:00 & 1998-03-25 16:15:15 & 1998-03-25 16:15:15 & 1998-03-25 16:22:05 & 132 & 0.06 & 0.04 \\
\hline 06 & 2000-10-11 04:15:00 & 2000-10-11 10:10:00 & 2000-10-11 10:10:00 & 2000-10-11 10:20:00 & 126 & 0.02 & 0.01 \\
\hline 07 & 2002-01-19 21:07:00 & 2002-01-19 21:45:00 & 2002-01-19 21:45:00 & 2002-01-19 21:46:00 & 108 & 0.14 & 0.17 \\
\hline 08 & 2002-04-09 20:25:20 & 2002-04-09 21:01:30 & 2002-04-09 20:24:30 & 2002-04-09 20:25:20 & 81 & 0.09 & 0.07 \\
\hline 09 & 2003-08-05 08:24:00 & 2003-08-05 10:23:30 & 2003-08-05 08:17:35 & 2003-08-05 08:24:00 & 112 & 0.02 & 0.01 \\
\hline
\end{tabular}

Notes. ${ }^{(a)}$ The code number of the interplanetary magnetic flux rope associated with reconnection exhaust. ${ }^{(b)}$ The front boundary of the interplanetary magnetic flux rope (UT). ${ }^{(c)}$ The rear boundary of the interplanetary magnetic flux rope (UT). ${ }^{(d)}$ The beginning of the reconnection exhaust (UT). ${ }^{(e)}$ The end of the reconnection exhaust (UT). ${ }^{(f)}$ The magnetic field shear angle across the reconnection exhaust (degree). ${ }^{(g)}$ The external proton beta near the front edge of the reconnection exhaust. ${ }^{(h)}$ The external proton beta near the rear edge of the reconnection exhaust.

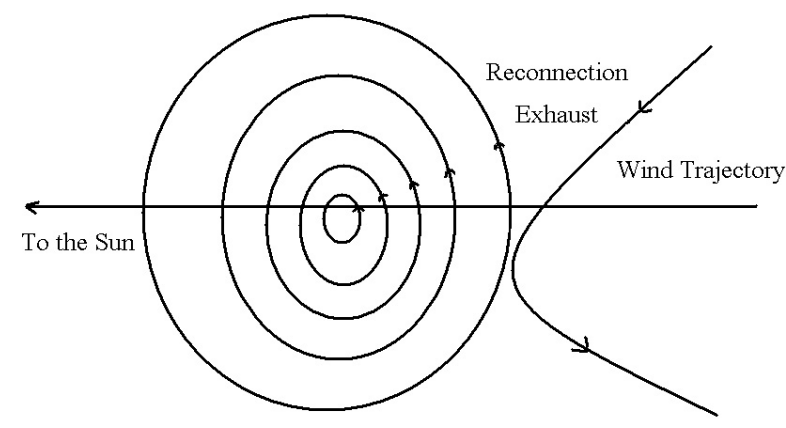

Fig. 3. Schematic sketch of he cross section of the flux rope and the reconnection exhaust along with the Wind trajectory through the event. We point out that Figs. 1 and 2 show an acceleration along the $x$ and $y$ axes, but very little acceleration was observed along the $z$ axis. This suggests that the exhaust is directed along the flux rope surface and outof-the plane of the paper. Figure 3 does not correctly reflect the data that it is based on.

to the fitting results, Fig. 3 shows the sectional sketch of the flux rope and the reconnection exhaust. The sketch suggests that the magnetic flux in the SIMFR is decreasing by the magnetic reconnection. Therefore, the spatial scale of the flux rope would diminish gradually.

Except for the six reconnection exhausts that occurred at the front boundaries of their related SIMFRs, the other three occurred at the rear boundaries of their related SIMFRs (see Table 1). As an example, Fig. 4 shows the SIMFR on 2002 January 19 as well as the trailing magnetic reconnection exhaust. Figure 4 shows an accelerated flow event that followed the SIMFR. During the accelerated flow, the proton density, temperature, and proton beta are all enhanced, whereas the field strength is depressed. All the above properties are characteristic of reconnection exhausts. In addition, Fig. 4 also reveals that the exhaust occurred within a low proton beta solar wind which has a slightly different plasma and magnetic field states on either side of the reconnection event. Figure 5 demonstrates that the changes in $\boldsymbol{V}$ and $\boldsymbol{B}$ were correlated with one another at the leading edge of the event and were anticorrelated at the trailing edge. Thses pairs of coupled changes in $\boldsymbol{V}$ and $\boldsymbol{B}$ are also the characteristic signature of reconnection exhausts in solar wind. Figure 4 shows that the reconnection exhaust and the SIMFR are juxtaposed, and no measurable separation exists between them. This means that reconnection was opening the flux rope fields and connecting them to the solar wind IMF, thus leading to a loss of flux.
Table 1 indicates that the timescale of these nine exhausts ranges from 1 to $10 \mathrm{~min}$. Table 1 also reveals the local magnetic field shears and external proton betas of the nine identified reconnection exhausts. The observed magnetic field shears across the exhausts range from $80^{\circ}$ to $154^{\circ}$, and the external proton betas range from 0.01 to 0.29 . As shown in Table 1 , the external proton betas on the opposite sides of the same exhaust are different. In addition, all reconnection exhausts are juxtaposed with their related SIMFRs, and no apparent gap is observed between the exhausts and their related SIMFRs. This suggests that all nine SIMFRs were being destroyed by reconnection. On the other hand, although the simulation result showed that magnetic reconnection may produce rope topology (Lee et al. 1993), in these cases the magnetic reconnection is destroying instead of generating the SIMFRs. If these SIMFRs were generated by magnetic reconnection, the SIMFRs would need to be immersed within the exhaust flow. However, the durations of the nine exhaust regions are too short compared with the dimensions of the SIMFRs. Therefore, although it is hard to obtain the evidence against or in favor of flow toward the flux ropes from single $\mathrm{X}$-line, the likelihood that the exhausts generated the adjacent SIMFRs is low. It is much more probable that the X-lines are the flux rope surface counterparts of magnetopause reconnection at Earth and that they indeed destroy some fraction of the flux rope.

As mentioned above, Wei et al. (2003) found that most magnetic clouds have boundary layers displaying a drop in the magnetic field magnitude and a significant change in the field direction as well as properties of a higher proton temperature, density and plasma beta. They argued that these boundary layers can possibly form through the magnetic reconnection process as a result of the interactions between magnetic clouds and the ambient solar wind. We analyzed the observational characteristics near the boundaries of the other 89 SIMFR events not related to the reconnection exhausts. The boundaries of most of these events also have a higher proton temperature, density, plasma beta, magnetic field depression, and shear angle. In our future work, we will conduct a detailed study of the boundary layers of SIMFRs. The boundaries of SIMFRs have properties similar to those of magnetic clouds in that both exhibit signatures of magnetic reconnection. Therefore, we speculate that magnetic reconnection may have occurred near the boundary before the Wind spacecraft observed these events, and the boundaries of most SIMFRs are still evolving through interaction with the background solar wind. As a result, their spatial scales would diminish gradually. 
H. Q. Feng et al.: Magnetic reconnection and flux rope

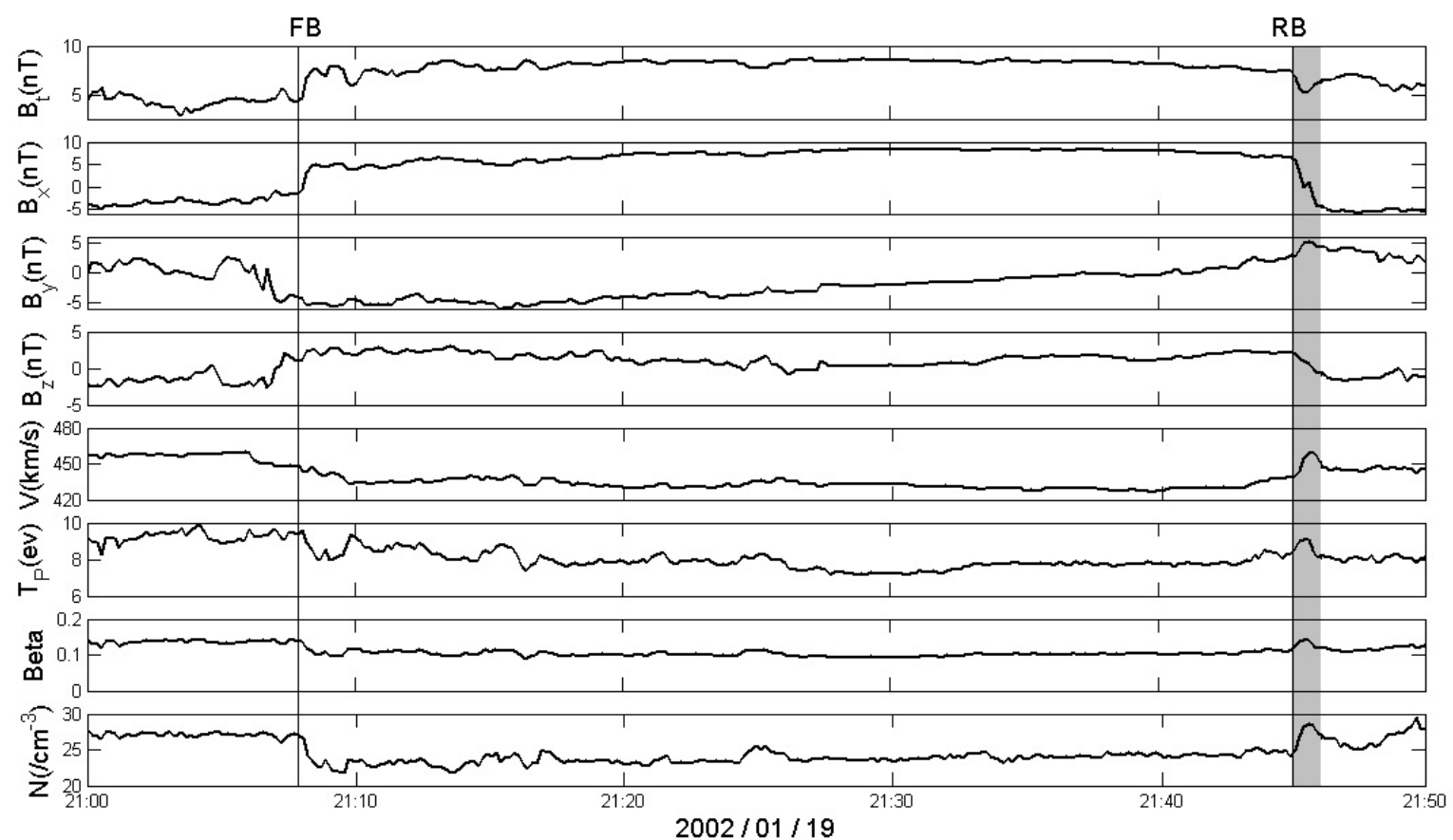

Fig. 4. Interplanetary magnetic field and plasma data measured by the Wind spacecraft on 2002 Jan. 19. The gray shaded region (21:45:0021:46:00 UT) indicates the magnetic reconnection exhaust. FB and RB are the front and rear boundaries of the interplanetary magnetic flux rope, respectively.

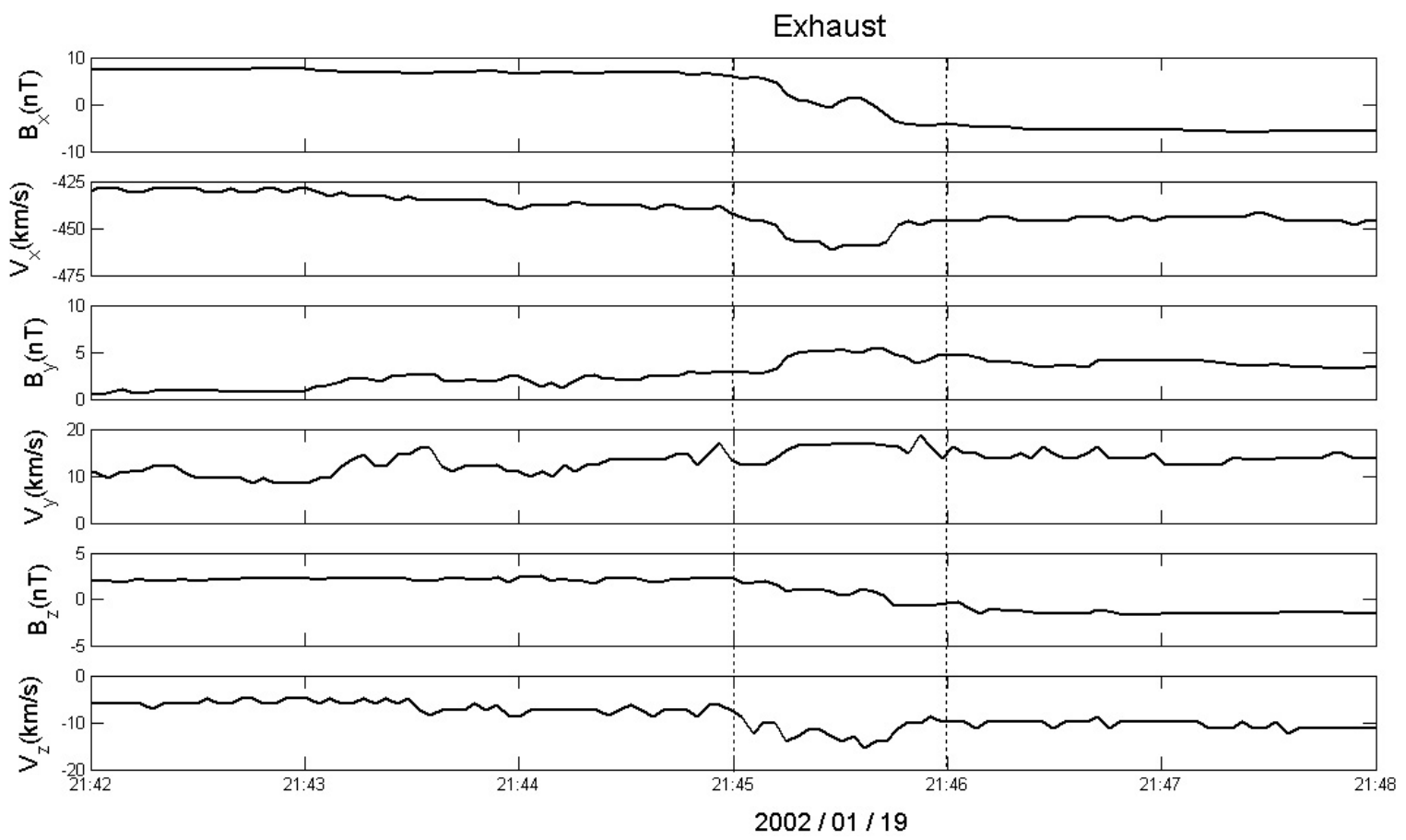

Fig. 5. Interplanetary magnetic field and speed data measured by the Wind spacecraft during the 2002 Jan. 19 magnetic reconnection exhaust passage. The dotted vertical lines indicate the beginning and the end of the reconnection exhaust.

Acknowledgements. This work is supported by National Nature Science Foundation of China (NSFC) under grant Nos. 41074124, 40804034 and 40890162. This work is also sponsored by Program for Science \& Technology Innovation Talents in Universities of Henan Province (HASTIT) under grant Nos. 2010HASTIT022, and by a Project Supported by the Specialized Research Fund for State Key Laboratories. This work is supported in part by Program for Science \& Technology of Henan under grant Nos. 092300410189. The authors thank NASA/GSFC for the use of data from Wind.

\section{References}

Belcher, J. W., \& Davis, L., Jr. 1971, J. Geophys. Res., 76, 3534

Bothmer, V., \& Schwenn, R. 1998, Ann. Geophys., 16,

Burlaga, L. F. 1988, J. Geophys. Res., 93, 7217

Burlaga, L. F., \& Turner, J. M. 1976, J. Geophys. Res., 81, 73

Burlaga, L. F., Lepping, R. P., \& Jones, J. A. 1990, in Physics of magnetic flux ropes (Washington, DC: American Geophysical Union), Geophysical

Monograph Ser., 58, 373 
Cartwright, M. L., \& Moldwin, M. B. 2008, J. Geophys. Res., 113, A09105 Coleman, P. J., Jr. 1967, Planet. Space Sci., 15, 953

Coleman, P. J., Jr. 1968, ApJ, 153, 371

Farrugia, C. J., Osherovich, V. A., \& Burlaga, L. F. 1995, J. Geophys. Res., 100, 12293

Feng, H. Q., \& Wu, D. J. 2009, ApJ, 705, 1385

Feng, H. Q., Wu, D. J., \& Chao, J. K. 2007, J. Geophys. Res., 112, A02102

Feng, H. Q., Wu, D. J., Lin, C. C., et al. 2008, J. Geophys. Res., 113, A12105

Feng, H. Q., Chao, J. K., Lyu, L. H., \& Lee, L. C. 2010, J. Geophys. Res., in press

Goldstein, H. 1983, NASA Conference Publication, 228, 731

Gosling, J. T., Skoug, R. M., McComas, D. J., \& Smith, C. W. 2005, J. Geophys. Res., 110, 1107

Gosling, J. T., Eriksson, S., Phan, T. D., et al. 2007, Geophys. Res. Lett., 34, 6102
Kahler, S. 1987, Sixth International Solar Wind Conference, 215

Hundhausen, A. J. 1987, Sixth International Solar Wind Conference, 181

Lee, L. C., \& Fu, Z. F. 1985, Geophys. Res. Lett., 12, 105

Lee, L. C., Ma, Z. W., Fu, Z. F., \& Otto, A. 1993, J. Geophys. Res., 98, 3943

Lepping, R. P., Burlaga, L. F., \& Jones, J. A. 1990, J. Geophys. Res., 95, 11957

Moldwin, M. B., Phillips, J. L., Gosling, J. T., et al. 1995, J. Geophys. Res., 100, 19903

Moldwin, M. B., Ford, S., Lepping, R., Slavin, J., \& Szabo, A. 2000, Geophys. Res. Lett., 27, 57

Petschek, H. E. 1964, NASA Special Publication, 50, 425

Webb, D. F., Lepping, R. P., Burlaga, L. F., et al. 2000, J. Geophys. Res., 105, 27251

Wei, F., Liu, R., Fan, Q., \& Feng, X. 2003, J. Geophys. Res., 108, 1263

Wu, C.-C., \& Lepping, R. P. 2002, J. Geophys. Res., 107, 1314 